\title{
A Decision Support System for Tomato Growers Based on Plant Responses and Energy Consumption
}

\author{
J. Hanssens, T. De Swaef and K. Steppe \\ Laboratory of Plant Ecology \\ Ghent University \\ Ghent
}

Belgium

H. Marien

Thomas More Kempen

Geel

Belgium

\author{
D. Pinxteren \\ Proefcentrum Hoogstraten \\ Meerle \\ Belgium
}

\author{
L. Wittemans \\ Proefstation voor de groenteteelt \\ Sint-Katelijne-Waver \\ Belgium
}

\section{J. Desmedt}

Flemish institute for technological research (VITO)

Mol

Belgium

Keywords: Solanum lycopersicum, mechanistic modelling, fruit growth, LVDT, greenhouse climate model, model calibration, model validation, energy

\begin{abstract}
The importance of plant water status for a good production and quality of tomato fruits (Solanum lycopersicum L.) has been emphasized by many authors. Currently, different new energy-saving technologies and growing strategies are under investigation to cope with the increasing fossil fuel prices. However, these technologies and growing strategies typically alter the greenhouse climate, thereby affecting the plants' response. Hence, the question arises how to adapt the microclimate to reduce the energy consumption of greenhouse tomato cultivation without compromising fruit yield or quality. Nowadays, the use of plant-based methods to steer the climate is of high interest and it was demonstrated that monitoring of stem diameter variations and fruit growth provides crucial information on both the plant water and carbon status. However, interpretation of these data is not straightforward and, hence, mechanistic modelling is necessary for an unambiguous interpretation of the dynamic plant response. During a 4-year research period, we investigated the response of different plant processes of tomato to dynamic microclimatic greenhouse conditions. The final aim was to develop a decision support system that helps growers to find an optimal balance between energy consumption, plant response and fruit yield. To this end, an integrated plant model, including stem, leaves, roots and fruits, was developed in which the various plant processes are mechanistically described. The plant model was calibrated and extensively validated on datasets collected throughout the different growing seasons in different research facilities in Flanders. This plant model was finally integrated into an existing greenhouse climate model and validated with data from the greenhouse climate and energy consumption. After validation, this integrated model
\end{abstract}


was used to run scenarios on growing strategies and their impact on energy consumption, plant photosynthesis and fruit growth.

\section{INTRODUCTION}

Tomato (Solanum lycopersicum L.) cultivation in Flanders, Belgium, is an energyconsuming activity. As fossil fuel prices continue to increase, various new energy-saving technologies and growing strategies are currently introduced. These new technologies and growing strategies influence the greenhouse climate, and, as such, affect the plants' response. Many authors have already emphasized the importance of plant water status in order to guarantee a good production and quality of tomato fruits (Mitchell et al., 1991; Johnson et al., 1992). Since both tomato yield and quality are of high importance in Flanders, it is of the utmost importance to maintain a proper plant water status under changing microclimatic conditions. Nowadays, the use of plant sensors for this purpose is of high interest: monitoring of stem diameter variations provides crucial information on both the water and carbon status of the plant (De Swaef et al., 2013). However, interpretation of these data can be difficult and, hence, mechanistic modelling is a prerequisite for an unambiguous interpretation of the dynamic plant response. De Swaef and Steppe (2010) adapted a mechanistic flow and storage model, originally developed for trees (Steppe et al., 2006). This model allows the simulation of stem diameter variations, xylem water potential and turgor pressure based on measurements of sap flow. Recently, the model of De Swaef and Steppe (2010) was successfully coupled to the biophysical model of Liu et al. (2007) to study the response of plant water status, stem diameter variations and fruit growth to elevated temperature and vapour pressure deficit (VPD) (Hanssens et al., 2012b).

During a 4-year research period, the response of different plant processes of tomato to dynamic microclimatic conditions in the greenhouse was studied. The final goal was the development of a decision support system that can help growers to find an optimal balance between energy consumption, plant response and fruit yield. Therefore, an integrated plant model, including plant processes at different levels (stem, leaves and fruits), was developed and the various plant processes were mechanistically described. The plant model was calibrated and extensively validated on datasets collected throughout the different growing seasons in two research facilities in Flanders (Hanssens et al., 2012a; Hanssens et al., 2012b). This plant model was finally integrated into an existing greenhouse climate model and validated with data from the greenhouse climate and energy consumption. After validation, this combined plant and greenhouse climate model was used to run scenarios on growing strategies and their impact on energy consumption, photosynthesis and fruit growth.

\section{MATERIALS AND METHODS}

\section{Microclimatic, Plant Physiological and Outdoor Climate Measurements}

During the growing seasons of 2009 till 2012, tomato plants were continuously monitored in different greenhouse compartments of two research facilities in Flanders, Belgium. Sap flow was measured below the first leaf using a heat balance sap flow sensor (Model SGA13-WS, Dynamax Inc., Houston, TX, USA; accuracy 10\%), installed according to the operation manual (van Bavel and van Bavel, 1990). Stem diameter variations and fruit growth were measured with linear variable displacement transducers (LVDT; Model 2.5 DF, Solartron Metrology, Bognor Regis, UK; accuracy $\pm 2.5 \mu \mathrm{m}$ ), attached using custom-made stainless steel holders. Leaf temperature was monitored with 
infrared temperature sensors (IRt/c.1X, Exergen Corporation, USA). Additionally, air temperature and relative humidity ( $\mathrm{T}_{\mathrm{a}}$ and $\mathrm{RH}$; Model HMP50, Vaisala, Finland) were continuously measured in the vicinity of the monitored plants. Finally, photosynthetically active radiation (PAR; Model JYP1000, SDEC, France) was measured above the canopy. All sensor signals were logged every 30 seconds using a data logger (CR1000, Campbell Scientific Inc., Logan, Utah, USA) and averaged every five minutes. The outside climate was registered with a weather station installed outside the greenhouse. A climate box (HortiMaX, the Netherlands) controlled the indoor greenhouse climate and data from control variables were logged.

During the 2012 growing season, photosynthesis and stomatal conductance at different levels within the canopy were monthly measured using a LI-6400XT (LiCor Biosciences, Lincoln, NE, USA). Furthermore, stem water potential was determined destructively using a pressure chamber (PMS Instrument Company, Corvallis, OR, USA) on leaves covered for $1 \mathrm{~h}$ with plastic bags coated with aluminum foil. These data were used to calibrate the different submodels in the plant model at different times of the growing season.

\section{Model Description, Calibration and Simulation}

For this study, the coupling between the flow and storage model of De Swaef and Steppe (2010) and the fruit model of Liu et al. (2007), was extended with a photosynthesis model based on the Farquhar, von Caemerer \& Berry model (Farquhar et al., 1980), which was calibrated and validated for tomato. A leaf model described the loading of assimilates from the leaves to the stem (De Swaef et al., 2013). In the original flow and storage model of De Swaef and Steppe (2010), sap flow data were used as input variable, but this input variable is now replaced by a transpiration model (Stanghellini, 1987), which calculates transpiration based on the greenhouse climate. Sap flow measurements, stomatal conductance data and measured leaf temperature served for calibration and validation of this transpiration model.

Next, the plant model was integrated in an existing greenhouse climate model (Bontsema et al., 2002). A tight coupling between both models was established at the level of crop transpiration, where a strong mutual influence between the energy balance of the greenhouse and the crop transpiration takes place.

Subsequently, the greenhouse model was made self-regulating by implementation of a proportional controller for the tube rail and growing tube temperature and the window opening. This proportional controller allowed adjusting these steering variables based on the difference between a setting and an actual value of temperature or relative humidity.

Finally, the energy consumption of the greenhouse was calculated from the flow of water through the heating tubes, the temperature difference between the ingoing and outgoing water and the density and heat capacity of water. This calculated energy use was then compared with the measured energy consumption.

Model implementation, model calibration and simulation were performed in PhytoSim (Phyto-IT, Mariakerke, Belgium). Calibration was carried out using the simplex method (Nelder and Mead, 1965) and a fourth-order Runge-Kutta numerical integrator with a fixed step size (0.01) was used for the simulations.

\section{RESULTS AND DISCUSSION Model validation}


Fig. 1 gives an overview of the outdoor climate on the $10^{\text {th }}$ and $11^{\text {th }}$ February 2012. The weather was very cold and sunny. In Fig. 2, the control variables are shown. The windows were kept close all day and the thermal screen (XLS10, Svensson, The Netherlands) was open between sunrise and sunset. High tube temperatures were required to reach the temperature settings in the greenhouse. Using the outdoor conditions of Fig. 1 and the control variables of Fig. 2, the combined plant and greenhouse climate model was used to predict the indoor conditions and plant response (Fig. 3). Simulated indoor temperature (Fig. 3A) and transpiration (Fig. 3C) corresponded well with measured data ( $\mathrm{R}^{2}$ of 0.89 and 0.87 , respectively). However, estimated RH (Fig. 3B) differed from the measurements, especially during daytime $\left(\mathrm{R}^{2}=0.61\right)$. As temperature and crop transpiration were well estimated, the overestimation of $\mathrm{RH}$ was probably the result from an underestimation of condensation on the greenhouse cover. A better implementation of this condensation process is crucial for good estimates of the $\mathrm{RH}$ in the greenhouse. Validation of the plant and greenhouse climate model for a period in May 2013 (results not shown) indicated that in conditions of higher outdoor temperatures, resulting in less condensation on the greenhouse cover, the $\mathrm{RH}$ in the greenhouse was predicted more accurately.

In Fig. 4, the performance of the self-regulating model is illustrated. Based on the outdoor climate (Fig. 1) and a desired indoor temperature (Fig. 4B, black solid line), the model adjusted tube temperatures to meet with the desired temperature settings. From Fig. 4, it can be seen that measured and simulated tube temperatures as well as indoor temperature showed close correspondence. The predicted and measured energy consumption of the greenhouse compartment also agreed well (Fig. 4C).

Finally, in Fig. 5, measured and simulated stem diameter (Fig. 5A) and fruit growth (Fig. 5B) are compared. The self-regulating model allowed simulating stem diameter variations and fruit growth based on the outdoor climate and desired climate settings. As such, this model can be used to analyse the plant response and energy use of a broad range of indoor climate settings in combination with different outdoor conditions. This way, it allows virtually testing various growing strategies, evaluating their effect on plant and energy and providing growers with a tool to find the optimal balance between energy cost and tomato fruit quality and production.

\section{Scenario Analysis}

After validation of the model, a first series of scenarios was run. Table 1 gives an overview of the effect of lowering the temperature settings on energy consumption, photosynthesis and production (increase of fruit fresh weight over a 24-h period), as estimated by the combined plant and greenhouse climate model. Decreasing the heating set point resulted in a marked decrease in energy consumption up till $13 \%$ when day and night temperature were lowered with $2^{\circ} \mathrm{C}$. Lowering the temperature settings during the night appeared to have a slightly larger impact on energy consumption. This might be explained by the very low night temperatures used for the scenario simulations requiring high tube temperatures to maintain the desired temperature, whereas during daytime, the incoming radiation provides an important source of energy. From Table 1, it is clear that the small temperature changes only have a minor impact on photosynthesis and production. This can be explained by the broad temperature optimum for photosynthesis of tomato plants. Temperature response curves of photosynthesis collected during the different growing seasons 
supported this (results not shown). However, it should be noted that these simulations reflect short term actions, since on the long term a lower temperature might affect plant balance. In Table 2, the effect of $\mathrm{CO}_{2}$ concentration in the greenhouse air was estimated for a sunny day in winter. Increasing the $\mathrm{CO}_{2}$ concentration from $400 \mathrm{ppm}$ to $700 \mathrm{ppm}$ resulted in a $30 \%$ increase of photosynthesis and a $6 \%$ increase in production. These results indicate that $\mathrm{CO}_{2}$ affects production to a much greater extent than temperature and emphasizes the importance of maintaining high $\mathrm{CO}_{2}$ levels in the greenhouse.

\section{CONCLUSIONS}

In this study, a mechanistic plant model and a greenhouse climate model were successfully coupled. Validation of this coupled model still revealed problems with the humidity balance of the greenhouse, especially during cold periods. The combination of greenhouse climate modelling and mechanistic models representing different plant processes show great potential for optimizing growing strategies and allow to virtually test various climate settings on their effect on energy consumption and plant response.

\section{ACKNOWLEDGEMENTS}

The authors are indebted to Philip Deman, Geert Favyts and Thomas Van De Putte for technical support and the IWT for funding granted to the first author. The authors would also like to thank LAVA, Priva and HortiMaX for financial support.

\section{Literature Cited}

Bontsema, J., Van Henten, E.J., de Gelder A., Janssen, H.J.J., Lamaker, A.J.J., van Meurs, W.T.M., Nijenhuis P., van Straten, G., Swinkels, G.L.A.M. and van Willigenburg, L.G. (2002) Optimaal energie efficiënt - model based besturingssysteem voor kasklimaat. Wageningen, IMAG B.V.: 75.

De Swaef, T. and Steppe, K. 2010. Linking stem diameter variations to sap flow, turgor and water potential in tomato. Funct. Plant Biol. 37:429-438.

De Swaef, T., Driever, S.M., Van Meulebroek, L., Vanhaecke, L., Marcelis, L.F.M. and Steppe, K. (2013) Understanding the effect of carbon status on stem diameter variations. Ann. Bot-London 111:31-46.

Farquhar, G.D., von Caemmerer, S. and Berry, J.A. (1980) A biochemical model of photosynthetic $\mathrm{CO}_{2}$ assimilation in leaves of $\mathrm{C}_{3}$ species. Planta 147:78-90.

Hanssens, J., De Swaef, T., Goen, K., De Nayer, F., Wittemans, L., Marien, H., Desmedt, J., Steppe, K. (2012a) Effect of stem age on the response of stem diameter variations to plant water status in tomato. Acta Hort. 952: 907-914.

Hanssens, J., De Swaef, T., Wittemans, L., Goen, K., Marien, H., Desmedt, J. and Steppe, K. (2012b) Model-assisted analysis of elevated temperature and vapour pressure deficit effects on tomato stem and fruit water balance. Acta Hort. 957:37-44.

Johnson, R.W., Dixon, M.A. and Lee, D.R. 1992. Water relations of the tomato during fruit growth. Plant, Cell and Environ. 15:947-953.

Liu, H.F., Génard, M., Guichard, S. and Bertin, N. 2007. Model-assisted analysis of tomato fruit growth in relation to carbon and water fluxes. J. Exp. Bot. 58:3567-3580.

Mitchell, J.P., Shennan, C. and Grattan, S.R. 1991. Developmental changes in tomato fruit composition in response to water deficit and salinity. Physiol. Plantarum 83:177185. 
Nelder, J.A. and Mead, R. 1965. A simplex method for function minimization. Comput. J. 7:308-313.

Stanghellini, C. (1987) Transpiration of greenhouse crops: an aid to climate management. $\mathrm{Ph} . \mathrm{D}$. dissertation, Agricultural University, Wageningen.

Steppe, K., De Pauw, D.J.W., Lemeur, R. and Vanrolleghem, P.A. 2006. A mathematical model linking tree sap flow dynamics to daily stem diameter fluctuations and radial stem growth. Tree Physiol. 26:257-273.

van Bavel, M.G. and van Bavel, C.H.M. 1990. Dynagage installation and operation manual. Dynamax Inc., Houston, TX.

\section{Tables}

Table 1. Effect of lowering the heating set point on energy consumption, photosynthesis and tomato fruit production over a 24-h period during cold and sunny winter conditions and a $\mathrm{CO}_{2}$ concentration of $400 \mathrm{ppm}$. Results are presented relative with respect to the reference situation (Fig. 3A).

\begin{tabular}{llll}
\hline Scenario & Energy & Photosynthesis & Production \\
\hline Reference & 100 & 100 & 100 \\
$\mathrm{~T}_{\text {day }}-1^{\circ} \mathrm{C}$ & 97.4 & 99.5 & 99.9 \\
$\mathrm{~T}_{\text {night }}-1^{\circ} \mathrm{C}$ & 96.2 & 100.6 & 100.2 \\
$\mathrm{~T}_{\text {day }}$ and $\mathrm{T}_{\text {night }}-1^{\circ} \mathrm{C}$ & 93.6 & 100 & 100 \\
$\mathrm{~T}_{\text {day }}-2^{\circ} \mathrm{C}$ & 94.3 & 98.9 & 99.8 \\
$\mathrm{~T}_{\text {night }}-2^{\circ} \mathrm{C}$ & 93.2 & 100.9 & 100.3 \\
$\mathrm{~T}_{\text {day }}$ and $\mathrm{T}_{\text {night }}-2^{\circ} \mathrm{C}$ & 87.3 & 99.8 & 100.1 \\
\hline
\end{tabular}

Table 2. Effect of increasing the $\mathrm{CO}_{2}$ concentration from $400 \mathrm{ppm}$ to $700 \mathrm{ppm}$ during cold sunny winter conditions. Results are presented relative with respect to the reference situation of $400 \mathrm{ppm}$.

\begin{tabular}{llll}
\hline Scenario & Energy & Photosynthesis & Production \\
\hline Reference & 100 & 100 & 100 \\
$700 \mathrm{ppm}$ & 100 & 129.6 & 106.1 \\
\hline
\end{tabular}




\section{Figures}

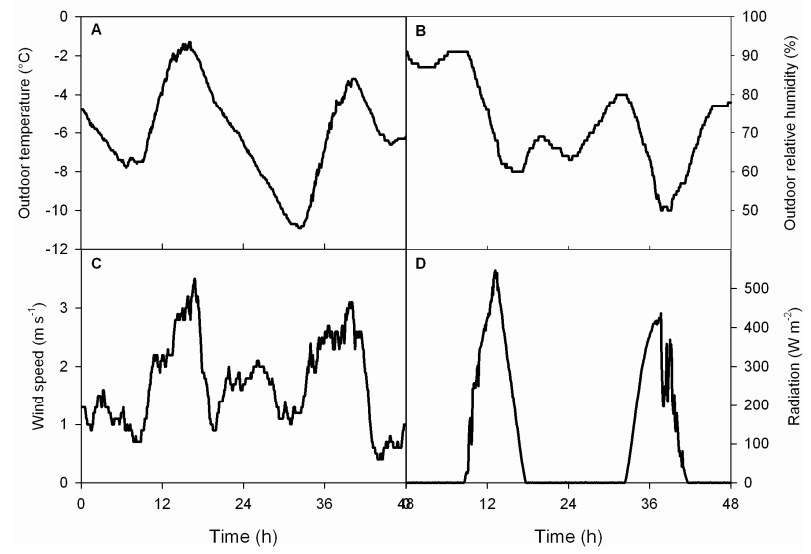

Fig.1. Outdoor climate on 10 and 11 February 2012: temperature (A), relative humidity (B), wind speed (C) and global radiation (D).

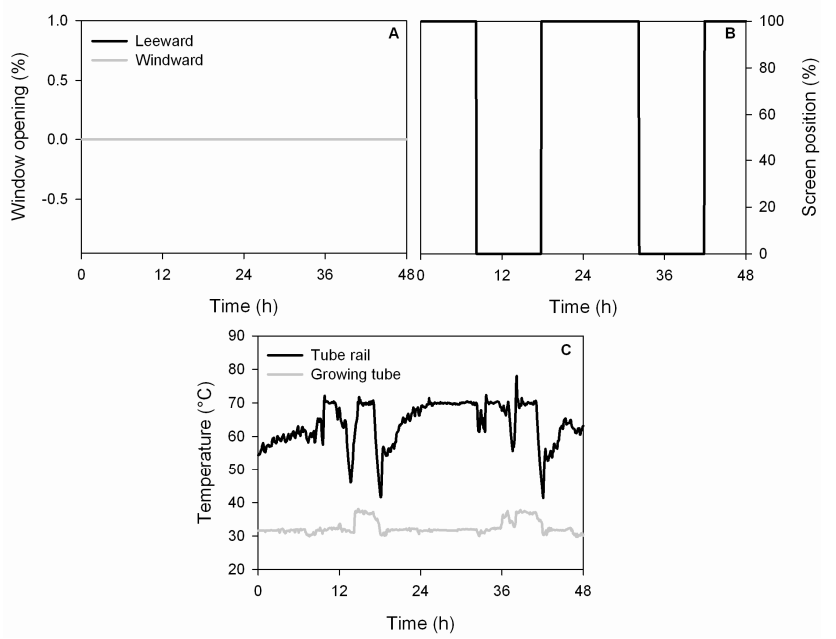

Fig. 2. Overview of the control variables on 10 and 11 February 2012: window opening (A), screen position (B) and tube temperatures (C).

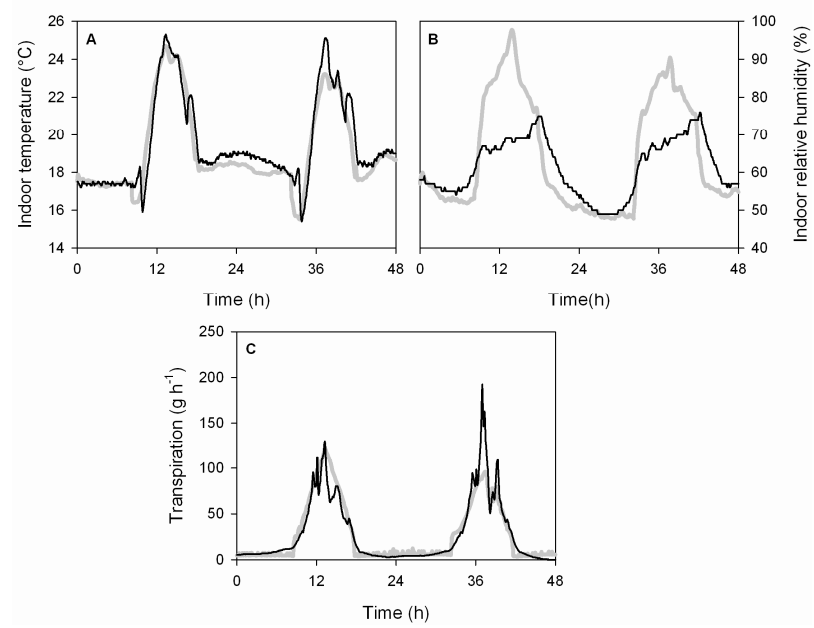

Fig. 3. Measured and simulated indoor temperature (A), indoor relative humidity (B) and transpiration (C) on 10 and 11 February 2012 using the original greenhouse climate model. 


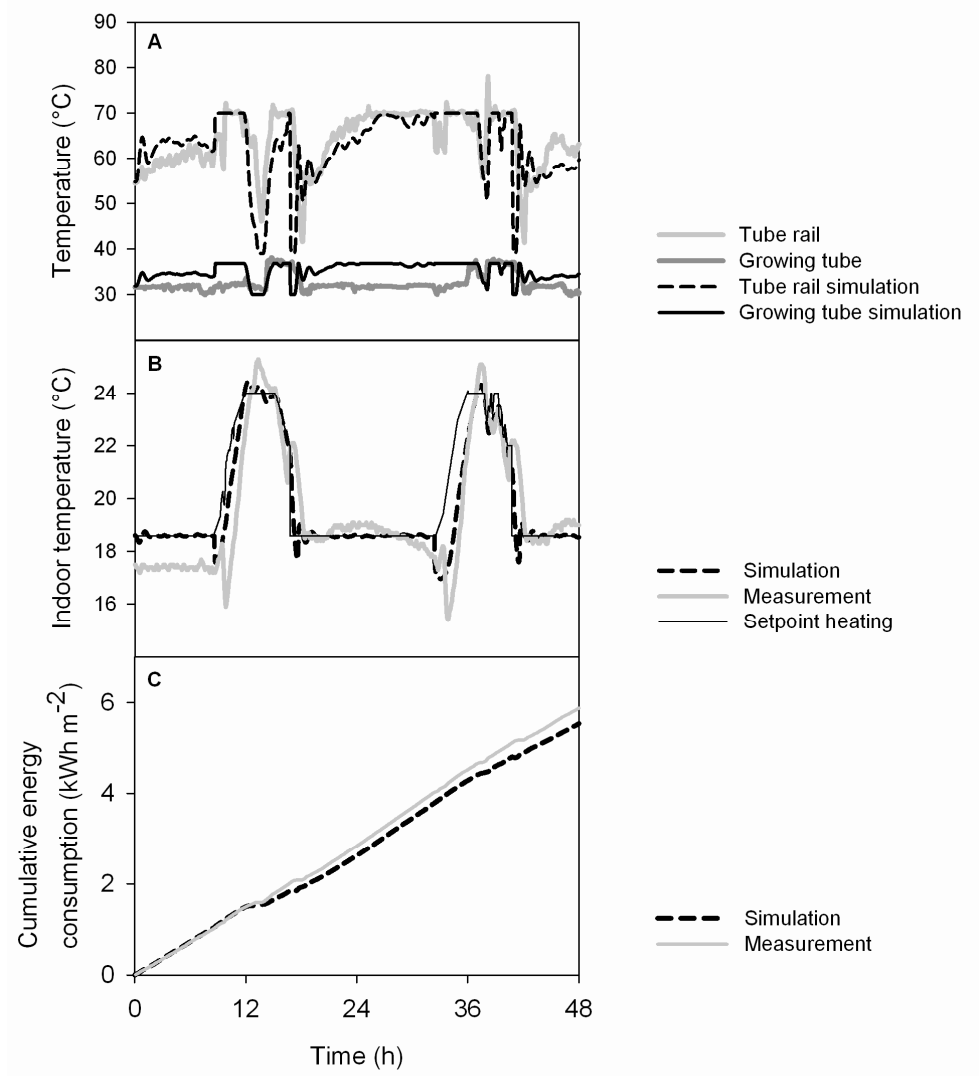

Fig. 4. Performance of the self-regulating plant and greenhouse climate model: measured and simulated tube temperatures (A), measured and simulated indoor temperature (B) and measured and predicted cumulative energy consumption (C) on 10 and 11 February 2012.

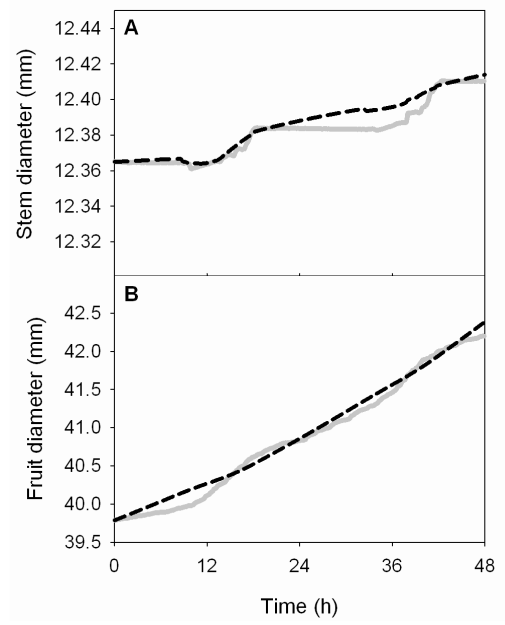

Fig. 5. Comparison between the measured and simulated stem diameter (A) and fruit diameter (B) of a tomato plant on 10 and 11 February 2012 using the simulated greenhouse climate as input. 